\title{
THE AXIAL ROTATION RHYTHM OF UPPER EXTREMITY
}

\author{
WeI-Ning Chang ${ }^{1,2}$, Hong-Wen Wu ${ }^{3}$, Yi-Wen Chang ${ }^{4}$, Fong-Chin Su${ }^{5}$, Yi-Chao Lu ${ }^{1}$, \\ ChIEN-Jen Hsu ${ }^{1}$, ChI-YING WONG ${ }^{1}$ \\ ${ }^{1}$ Department of Orthopaedics, Kaohsiung Veterans General Hospital, Kaohsiung, Taiwan \\ ${ }^{2}$ Department of Surgery, School of Medicine, National Yang-Ming University, Taipei, Taiwan \\ ${ }^{3}$ School of Sports Medicine, Chinese Medical University, \\ ${ }^{4}$ Department of Exercise and Health Science, National Taiwan College of Physical Education, \\ Taichung, Taiwan \\ ${ }^{5}$ Institute of Biomedical Engineering, National Cheng Kung University, Tainan, Taiwan
}

\begin{abstract}
To determine the dynamic axial rotation movement of human upper extremity, we developed a combined skin-and scapula-based marker system in which the scapula motion was detected with a marker set attached to an intracortical pin on the acromion. The subject performed axial rotation with the arm in 5 different positions. The total rotation range varied greatly if the arm was in different position. The pattern of axial rotation of each segment also changed. The upper extremity rotates total $376^{\circ}$ at $90^{\circ}$ abduction and only $257^{\circ}$ at full abduction. The forearm usually contributed about $140^{\circ}$, so the differences mainly came from the scapulothoracic and the glenohumeral joints. The scapulothoracic joint axially rotated $66^{\circ}$ with the arm in backward extension while only rotated $19^{\circ}$ with the arm in $90^{\circ}$ forward flexion. The glenohumeral joint rotated maximally, $143^{\circ}$ with the arm at $90^{\circ}$ abduction, while minimally, $69^{\circ}$ with the arm at maximal abduction.
\end{abstract}

Biomed Eng Appl Basis Comm, 2005(December); 17: 275-280.

Keywords: axial rotation, shoulder, scapula, humerus, forearm, motion analysis

\section{INTRODUCTION}

Axial rotation is one of the major components of the upper limb motion. The rotation of an outreached arm is frequently used in the activities of daily living, work place, and in performance arts. The axial rotation movement of the shoulder and elbow (forearm

Received: July 21, 2005; Accepted: October 24, 2005 Correspondence: Fong-Chin Su, Professor

Institute of Biomedical Engineering, National Cheng Kung University, 1 University Rd., Tainan, Taiwan 701 E-mail: fcsu@mail.ncku.edu.tw rotation) had been widely studied in the literature. [1-9, 11-12] The forearm rotation is a composite motion occurring at the proximal and distal radioulnar joints, as well as the radiohumeral joint. The forearm axially rotates 68-75 degrees of pronation and 74-93 degrees of supination, with a total rotation range of 142-159 degrees (Table I ) $[2,5,7,10-11]$. To measure the forearm rotation, the upper arm is usually stabilized against the chest wall, and the elbow is flexed to 90 degrees [4]. The shoulder actively rotates 42-110 degrees in internal direction and 65-126 degrees in external direction with a total arc 113-193 degrees 
(Table II ) [1-3,5-6,8,10]. In above-mentioned studies, the rotation data of the shoulder and the forearm were not collected simultaneously, thus the rhythm between the upper arm and the forearm remains unknown. And because the scapula is a triangular bone covered with thick soft tissue, the bony contour of the scapula is difficult to palpate and the skeletal motion of the scapula is difficult to track. Many of the shoulder movement studies assumed the scapula and the humerus as one single segment and measured the scapulothoracic and the glenohumeral joint altogether. $[1-3,5-6,8,10]$. The purpose of this study was to propose a combined skin- and scapula-based marker system to investigate the active dynamic axial rotation motion of the scapulothoracic joint, the glenohumeral joint and the forearm simultaneously in living human subject.

\section{METHOD}

One healthy male subject exhibiting no upper extremity pathology participated in this study. The subject was 24 years old with body height $180 \mathrm{~cm}$ and body weight $72 \mathrm{~kg}$. The procedures employed were approved for use on human subjects by the Institutional Review Board. Before the experiment began, the subject signed an informed consent form that included an explanation of the procedures and a description of the experimental protocol.

We developed a 3-demensional biomechanical model of the upper extremity using combined skin and scapula-based marker sets. Four-segment link system was used to model the upper limb including the trunk, scapula, humerus, and forearm. To reduce the influence of undesirable skin marker motion of humerus and forearm, two orthosis were made, one for the elbow and one for the wrist. The elbow orthosis kept the elbow at full extension and molding was made to fit bony prominences of the medial, lateral epicondyle and the olecranon process. The wrist orthosis holded the wrist at neutral flexion/extension position. Cutting on

Table I . Rotation range of the forearm reported in the literature.

\begin{tabular}{lccc}
\hline $\begin{array}{c}\text { Pronation } \\
\text { (degree) }\end{array}$ & $\begin{array}{c}\text { Supination } \\
(\text { degree })\end{array}$ & $\begin{array}{c}\text { Total } \\
\text { (degree) }\end{array}$ & Source \\
\hline 71 & 84 & 155 & AAOS 1965 \\
71.2 & 88.0 & 159 & Wagner 1977 \\
75.8 & 82.1 & 158 & Boone 1979 \\
68 & 74 & 142 & Morrey 1996 \\
-- & $86.5-93.0$ & -- & Günal 1996 \\
\hline
\end{tabular}

distal radioulnar joint was made to allow full pronation/supination of forearm while restricting flexion/extension of the wrist. The orthosis was also useful to exclude the rotation of the carpus from the recording. We used invasive intracortical pinning in acromion to mark the scapula segment. Each segment was attached with 3 retroreflective markers. The marker placement was illustrated on Figure 1; the coordinate systems were defined as follows:

Trunk coordinate system: The z-axis was defined as the unit vector from PX to IJ. The y-axis was defined as the unit vector of the crossproduct of the $\mathrm{z}$-axis and the vector from $\mathrm{C} 7$ to IJ. The $\mathrm{x}$-axis was defined as the unit vector of the crossproduct of the $y$ - and z-axis. The transverse, frontal, and sagittal planes were defined accordingly.

Scapula coordinate system: The z-axis was defined as the unit vector from $\mathrm{AC}$ to $\mathrm{A} 3$. The $\mathrm{x}$-axis was defined as the unit vector of the crossproduct of

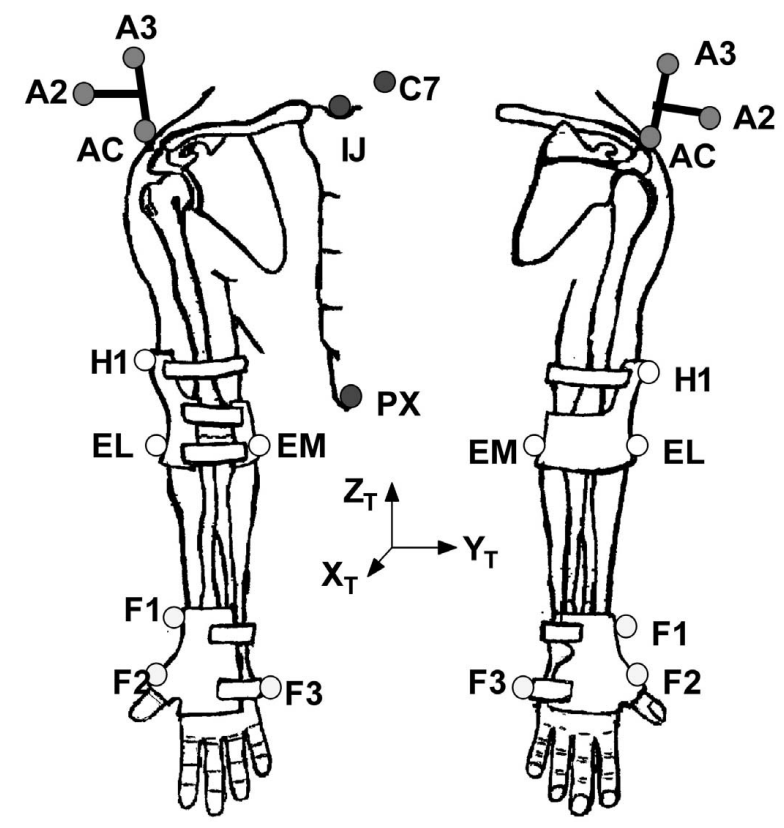

Fig 1. The four-segment system and the marker placement. 1. Trunk: PX: processus xyphoideus, IJ: incisura jugularis, $C 7: 7^{\text {th }}$ cervical vertebra. 2 . Scapula: AC: the $1^{\text {st }}$ marker of the cluster, A2: the $2^{\text {nd }}$ marker of the cluster, A3: the $3^{\text {rd }}$ marker of the cluster. 3. Humerus: EM: epicondylus medialis on the elbow orthosis, EL: epicondylus lateralis on the elbow orthosis, H1: lateral aspect of the elbow orthosis near the angulus inferior of the deltoid muscle. 4. Forearm: F1: radial aspect of proximal portion of the wrist orthosis, F2: radial aspect of distal portion of the wrist orthosis, F3: ulnar aspect of distal portion of the wrist orthosis. 
Table $\Pi$. Rotation range of the shoulder reported in the literature.

\begin{tabular}{lcccc}
\hline Arm position & $\begin{array}{c}\text { Internal } \\
\text { (degree) }\end{array}$ & $\begin{array}{c}\text { External } \\
(\text { degree })\end{array}$ & $\begin{array}{c}\text { Total } \\
(\text { degree })\end{array}$ & Source \\
\hline Abduction & $\sim 90$ & $\sim 90$ & $\sim 180$ & AAOS 1965 \\
$90^{\circ}$ abduction & 69 & 104 & 173 & Boone 1979 \\
$90^{\circ}$ abduction & $49-59$ & $82-101$ & $141-154$ & Murray 1985 \\
$90^{\circ}$ abduction & -- & -- & $111-149$ & Bonci 1986 \\
$90^{\circ}$ abduction & $45-60$ & $102-105$ & $149-164$ & Ellenbecker 1996 \\
-- & $95.5-110.4$ & $65.9-75.2$ & -- & Gunal 1996 \\
$90^{\circ}$ abduction & $39-75$ & $111-129$ & $162-194$ & Kibler 1996 \\
\hline
\end{tabular}

the $\mathrm{z}$-axis and the vector from $\mathrm{AC}$ to $\mathrm{A} 2$. The $\mathrm{y}$-axis was defined as the unit vector of the crossproduct of the $\mathrm{z}$ - and $\mathrm{x}$-axis.

Upper arm coordinate system: The y-axis was defined as the unit vector from the EL to EM. The $\mathrm{x}$ axis was defined as the unit vector of the crossproduct of y-axis and the vector from EL to H1. The z-axis was defined as the unit vector of the crossproduct of the $\mathrm{x}$ and y-axes.

Forearm coordinate system: The y-axis was defined as the unit vector from the F2 to F3. The $\mathrm{x}$-axis was defined as the unit vector of the crossproduct of $y$ axis and the vector from F2 to F1. The z-axis was defined as the unit vector of the crossproduct of the $\mathrm{x}$ and y-axes.

In this model, the y-axis (lateral to medial) of each segment represented the axis for flexion/extension; the $\mathrm{x}$-axis (posterior to anterior) represented the axis for adduction/abduction. The z-axis (verticle) represented the axis for segmental axial rotation.

The subject was instructed to perform the rotation movement of upper limb at a self-selected speed from the neutral position toward the internal direction until the extreme was reached, then rotated back to the external extreme. The subject did the axial rotation motion with the arm at 5 different positions after warm up exercise. The arm positions were:

(1) Rest position with the arm by side of the trunk: The thumb pointed anterior as the starting neutral position.

(2) Abduction 90 degrees: The arm elevates 90 degrees in frontal plane till was perpendicular to the trunk. The thumb pointed anterior as the starting neutral position.

(3) Maximal abduction: The arm abducted to maximum till it was parallel to the trunk. The thumb directed medially as the starting neutral position.

(4) Forward flexion 90 degrees: The arm flexed forward 90 degrees in sagittal plane till it was perpendicular to the trunk. The thumb directed upward as the starting neutral position.

(5) Backward extension to maximal extent: The arm extended backward in sagittal plane as much as possible without bending the trunk (about 45 degrees of backward extension). The thumb directed anterior as the starting neutral position.

The sequence of rotation is from neutral position to extreme internal rotation, then from extreme internal rotation to extreme external rotation. The images of the markers were collected by six video cameras and digitized by Expert Vision motion analysis system (Motion Analysis Corp., CA, USA). The axial rotation angle was calculated from the rotation angle of the projected y-axis unit vector of each segment on the trunk target plane, that was, transverse plane for resting postion, sagittal plane for $90^{\circ}$ abduction, transverse plane for maximum abduction, frontal plane for $90^{\circ}$ forward flexion, and $45^{\circ}$ anterior-tilted frontal plane for maximun backward extension. The value of axial rotation angle at a steady state before starting the rotation movement was defined as zero rotation angle of each arm postion. The calculation was performed with a custom MATLAB (MathWorks, MA, USA) program.

In the following text, the motions of distal segments relative to proximal segments describe the motions of the joints. The "forearm rotation" describes the motions from the proximal/distal radioulnar, and the radiocapitellar joints, is calculated from the rotation of the forearm coordinate system relative to the humerus coordinate system.

\section{RESULT}

The rotation ranges of the scapula, humerus and forearm and their cumulative rotation angles of arm at different positions were shown in Table III and Figure 
2. The dynamic axial rotation of the scapulothoracic joint, the glenohumeral joint and the forearm with reference to the trunk and its proximal segment were illustrated in Figures 3-7. The left plot of each figure demonstrated the rotation angles of each segment relative to the trunk. On the right two plots of each figure, the rotational motion was decomposed to represent individual motions of each of these 3 segments relative to its next proximal segment. The rotation ratio between each segment is different at the same position of rotation arc if the rotation direction is reversed. The initiation and the progression pattern of each segment are also different between five different arm positions.

Rest position: The humerus to forearm rotation ratio ranges from 1 to 1.2 from neutral to internal rotation. But the ratio is changed while the arm rotates back to neutral. The total rotation angle measured about 298 degrees; the contribution ratio of each segment is $7 \%, 44 \%$ and $49 \%$ for the scapula, humerus and forearm, respectively (Figure 3 ).

$90^{\circ}$ abduction: It is obvious that the humerus contributed more in internal direction and the forearm in external direction. The total arc measured 355 degrees with individual contribution $17 \%, 40 \%$, and $42 \%$ for the scapula, humerus and forearm, respectively (Figure 4). This is the position that allows the largest amount of rotation to happen between these five arm positions (Table III).

Maximal abduction: In this position, the humerus is somehow "locked" and only contributes 69 degrees in the 257 degrees total arc. The forearm rotates 141 degrees, which is a relatively constant amount in all of the arm positions (Figure 5).

$90^{\circ}$ forward flexion: Just like the condition in rest position, the scapula is somehow "locked" and contributes only 19 degrees to the total arc (Figure 6).

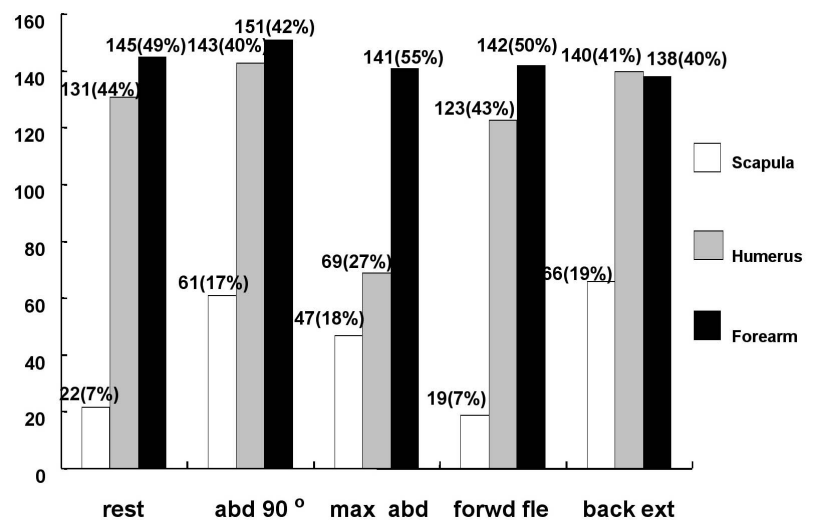

Fig 2. The rotation range and the percentage of total rotation of the scapula, humerus and forearm at 5 different arm positions.

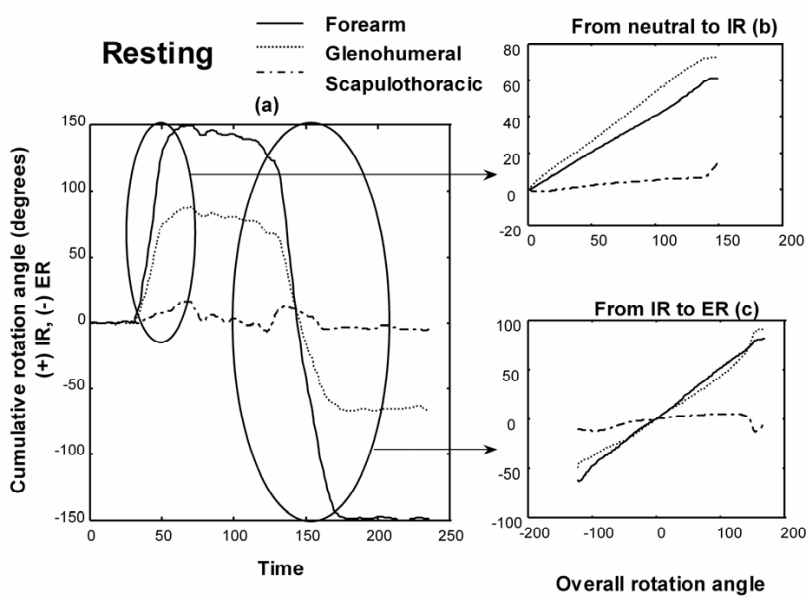

Fig 3. Axial rotation with the arm at resting position beside the trunk. The left plot (a) demonstrates the rotation angles of each segment "relative to the trunk". On the right two plots (b and c), the rotational motion was decomposed to represent individual motions of each of these 3 segments "relative to its next proximal segment" . Figures 4-7 were illustrated in the same manner. IR: internal rotation, ER: external rotation.
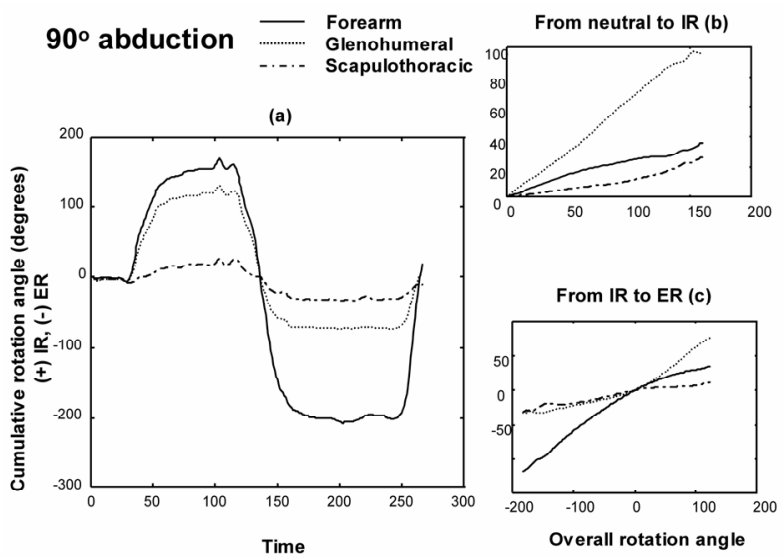

Fig 4. Axial rotation with the arm at 90 degrees abduction.

Backward extension: As shown in Figure 7, the forearm contributes most of the range of motion in the internal direction and the humerus in external direction. The condition is just opposite to that in 90 degrees abduction position. 
Table III . Rotation range (degrees) of the scapulothoracic joint, glenohumeral joint, and the forearm at 5 different arm positions.

\begin{tabular}{lccccc}
\hline & Rest & $\begin{array}{c}90^{\circ} \\
\text { abduction }\end{array}$ & $\begin{array}{c}\text { Maximum } \\
\text { abduction }\end{array}$ & $\begin{array}{c}\text { Forward } \\
\text { flexion }\end{array}$ & $\begin{array}{c}\text { Backward } \\
\text { extension }\end{array}$ \\
\hline Scapulothoracic & 22 & 61 & 47 & 19 & 66 \\
Glenohumeral & 131 & 143 & 69 & 123 & 140 \\
Forearm & 145 & 151 & 141 & 142 & 138 \\
\hline Total & 298 & 355 & 257 & 284 & 344 \\
\hline
\end{tabular}
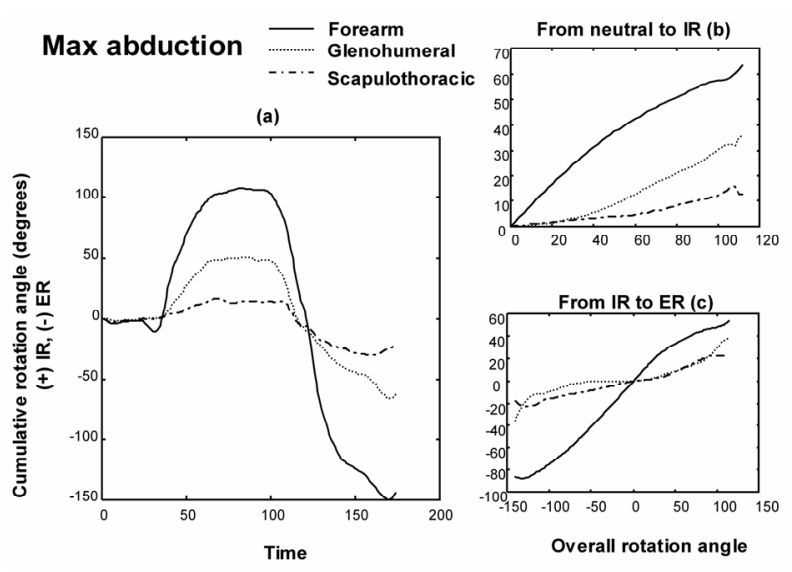

Fig 5. Axial rotation with the arm at maximum abduction.

\section{DISCUSSION}

The overall axial rotation angles of the arm varied greatly from 257 to 355 degrees with the arm at five different positions (Table III and Figure 2). The forearm constantly contributed about 140 (138-151) degrees during rotation, so the difference mainly came from the scapulothoracic and the glenohumeral joints. The scapulothoracic joint axially rotated $66^{\circ}$ with the arm in backward extension while only $19^{\circ}$ with the arm at $90^{\circ}$ forward flexion and 22 degrees at rest position. At these two positions, the scapula was fixed in a relatively stable position that limited its range of rotation. The glenohumeral rotation range was also varied with the arm in different positions. The glenohumeral joint rotated maximally (143 degrees) with the arm at 90 degrees abduction. The most limited rotation (69 degrees) was found when the arm was elevated to maximum. From the functional anatomy point of view, the reason of limited rotation might be caused by tightening of soft tissue or change of lever arm/action direction of musculature around the shoulder joint when the arm was elevated.
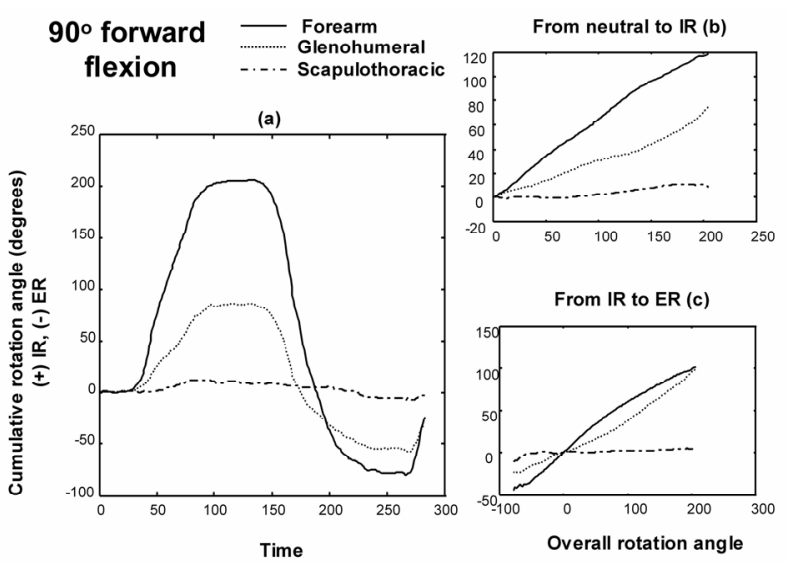

Fig 6. Axial rotation with the arm at 90 degrees forward flexion.
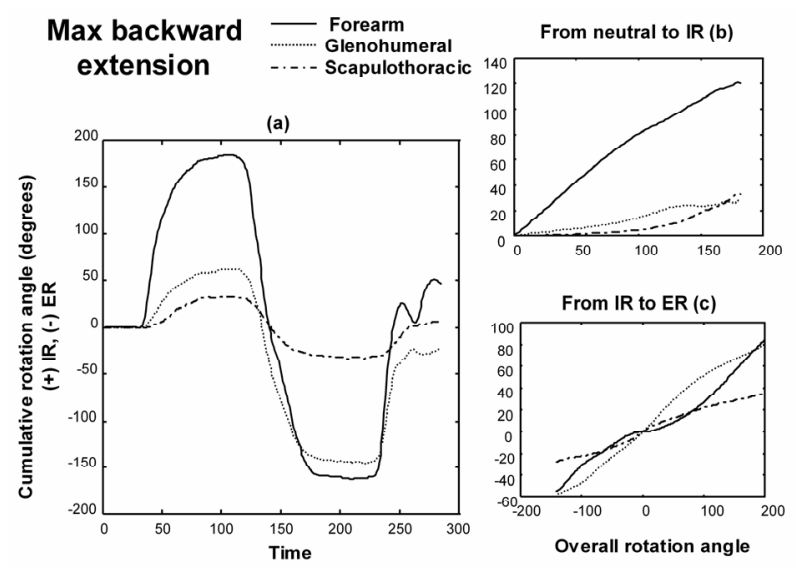

Fig 7. Axial rotation with the arm at maximum backward extension

Many other factors are also involved in the rotation range of motion of the shoulder including the subject's hand dominance $[1,3,5,6]$, the skeletal pathology[12], the voluntary control of the arm [9], the activity preference $[1,6]$, the gender $[1,3,6]$, and the 
age $[2,6]$.

In conclusion, the motion pattern, relationship, and contribution of each of the three joints of the upper extremity during axial rotation of the arm were studied using the skeletal marker of the scapula and the videobased motion analysis system. We determined the axial rotation rhythm of the upper extremity including the scapulothoracic joint, the glenohumeral joint, and the forearm in one subject. The interrelationship between each segment was dynamic and complex. In this study, it depended upon the arm position, at what range of rotation, and the direction of rotation. There was no constant "axial rotation rhythm" between three joints of the upper limb. This information, not previously recorded, may be useful to provide an objective basis for the design of occupational environment, to predict the rotation function remained after an upper limb arthrodesis, and to assist in the understanding of neuromuscular control of the upper extremity. This combined skin- and scapula-based marker system, successfully monitored the skeletal motion of the scapula relative to other segments, could be applied in future upper limb motion analysis studies.

\section{REFERENCE}

1. Bonci CM, Hensal FJ, Torg JS: A preliminary study on the measurement of static and dynamic motion at the glenohumeral joint. Am J Sports Med 1986; 14(1): 12-17.

2. Boone DC, Azen SP: Normal range of motion of joints in male subjects. J Bone Joint Surg Am 1979;61(5):756-59.

3. Ellenbecker TS, Roetert EP, Piorkowski PA, Schulz DA: Glenohumeral joint internal and external rotation range of motion in elite junior tennis players. J Orthop Sports Phys Ther 1996;24(6):336-41.

4. Greene WB, Heckman JD. The shoulder. In: The Clinical Measurement of Joint Motion. Rosemont: Am Academy Orthop Surgeons, 1994. 15-26.

5. Günal I, Kose N, Erdogan O, Gokturk E, Seber S: Normal range of motion of the joints of the upper extremity in male subjects, with special reference to side. J Bone Joint Surg 1996;78(9):1401-04.

6. Kibler WB, Chandler TJ, Livingston BP, Roetert EP: Shoulder range of motion in elite tennis players. Am J Sports Med 1996;24(3):279-285.

7. Morrey BF, Askew LJ, Chao EY: A biomechanical study of normal functional elbow motion. J Bone Joint Surg Am 1981;63(6):872-77.

8. Murray MP, Gore DR, Gardner GM, Mollinger LA: Shoulder motion and muscle strength of normal men and women in two age groups. Clin
Orthop 1985;192:268-73.

9. Perry J: Normal upper extremity kinesiology. Phys Ther 1978;58(3):265-278.

10. The Committee for the Study of Joint Motion (ed). Joint motion: Method of measuring and recording. Chicago, IL, Am Academy Orthop Surgeons, 1965.

11. Wagner C: Determination of the rotary flexibility of the elbow joint. Eur J Appl physiol Occup Physiol 1977;37(1):47-59.

12. Warner JJP, Micheli LJ, Arslanian LE, Kennedy J, Kennedy R: Patterns of flexibility, laxity and strength in normal shoulders and shoulders with instability and impingement. Am J Sports Med 1990;18: 366-375. 